\title{
Biodegradation and biotransformation of pentachlorophenol by wood-decaying white rot fungus Phlebia acanthocystis TMIC34875
}

Pengfei Xiao ${ }^{1 *}$ (1) and Ryuichiro Kondo ${ }^{2}$

\begin{abstract}
Pentachlorophenol (PCP) has been introduced into the environment mainly as a wood preservative and biocide. The degradation and transformation of PCP in liquid culture by wood-decaying fungus capable of degrading organochlorine pesticides was investigated in this study. The results of tolerance test showed that the tolerance level of Phlebia acanthocystis to PCP in potato dextrose agar medium was higher than that of other Phlebia species. At the end of 10 days of incubation, P. acanthocystis was able to remove $100 \%$ and $76 \%$ of PCP $(25 \mu \mathrm{M})$ in low-nitrogen and potato dextrose broth media, respectively. The decrease of PCP in P. acanthocystis culture is accompanied by the formation of pentachloroanisole and $p$-tetrachlorohydroquinone via methylation and oxidation reactions. Moreover, the $p$-tetrachlorohydroquinone formed is rapidly converted to methylated products including tetrachloro-4-methoxyphenol and tetrachloro-1,4-dimethoxybenzene. The activities of lignin peroxidase and manganese peroxidase were found to increase in extracellular fluid from fungal culture treated with high-concentration PCP, with maximum values of 169.6 $\mathrm{U} / \mathrm{L}$ and $73.4 \mathrm{U} / \mathrm{L}$, respectively. The in vitro degradation of $\mathrm{PCP}$ and $p$-tetrachlorohydroquinone was confirmed using extracellular fluid of $P$. acanthocystis, suggested that the methylation of both compounds is related to extracellular enzymes. Degradation of PCP was efficiently inhibited by piperonyl butoxide or 1-aminobenzotriazole, demonstrating that cytochrome P450 monooxygenase is involved in fungal transformation of PCP, particularly in the oxidation of PCP to $p$-tetrachlorohydroquinone. Additionally, P. acanthocystis mineralized $9.3 \%$ of the PCP to ${ }^{14} \mathrm{CO}_{2}$ in low-nitrogen culture during 42 days. Results obtained in the present study are in favor of the use of $P$. acanthocystis as a microbial tool of remediation of PCP-contaminated sites.
\end{abstract}

Keywords: Biodegradation, Biotransformation, Pentachlorophenol, Phlebia acanthocystis, Ligninolytic enzymes, Cytochrome P450 monooxygenase

\section{Introduction}

Pentachlorophenol (PCP) was widely used since the 1930s as a wood preservative and biocide in industry and agriculture around the world, due to its broad spectrum and low cost. Substantial amounts of sodium pentachlorophenol salts have been sprayed in central China since the 1960s for control of snail-borne schistosomiasis. This generalized use of PCP has led to the contamination of

\footnotetext{
*Correspondence: xpfawd@nefu.edu.cn

${ }^{1}$ College of Forestry, Northeast Forestry University, Hexing Road 26, Harbin 150040, China

Full list of author information is available at the end of the article
}

water and soil systems, with PCP currently considered to be a product of priority for decontamination studies [1]. Although the application of PCP in agriculture has been banned since 2000, it is recently still detected in the environment around China due to its molecular stability and sorption properties [2]. PCP has been reported to be carcinogenic, mutagenic, causes acute pancreatitis, acts as endocrinal agents, and is generally a significant toxic element to humans [3].

Given the potential hazards associated with $\mathrm{PCP}$, an efficient method for remediation is required, while for wood rot fungi, a future in bioremediation is definitely possible, because they are not dangerous to humans or 
animals. These fungi occur naturally growing on wood, and have demonstrated the ability to degrade a wide range of recalcitrant environmental pollutants. Wood rot fungi are known to secrete non-specific enzymes, and therefore they are generally more tolerant to a large diversity of, and high concentrations of a toxic pollutant than other microorganisms, which need to uptake the toxic pollutant in order to degrade it. Extracellular ligninolytic enzymes and cytochrome P450 enzymes of wood rot fungi have been reported to be responsible for organic pollutants degradation. Phanerochaete chrysosporium [4], Phanerochaete sordida [5], Trametes hirsuta [6], Trametes versicolor [7], Lentinula edodes [8], Bjerkandera adusta [9], Anthracophyllum discolor [10], Laetiporus cincinnatus, Fomes fomentarius, Ganoderma applantum and Pleurotus ostreatus [11] are some of the wood rot fungi that show the ability to degrade PCP, and methylation and dechlorination are common reactions in transformation of PCP by these fungi. Among them, $P$. chrysosporium and $T$. versicolor have been the most widely used wood rot fungi for PCP degradation. Nevertheless, the metabolic pathway and enzymatic mechanism for fungal degradation of PCP have not been clarified, a possible reason attributed to the high degree of chlorination in PCP molecule, and involvement of complex and nonspecific enzyme systems.

In our previous study, wood rot fungi belonging to the genus Phlebia were evaluated based on their ability to degrade organochlorine pesticides such as heptachlor, dieldrin and aldrin, and found that most of these Phlebia strains were able to degrade these compounds in pure culture, and Phlebia acanthocystis was most effective in degrading organochlorine pesticides among 20 species of Phlebia [12, 13]. Moreover, it has been demonstrated in these studies that these recalcitrant compounds were transformed to hydrophilic products via hydrolysis, dechlorination and hydroxylation by Phlebia strains. Subsequently, Purnomo et al. [14] compared the ability of several wood rot fungi to degrade heptachlor and heptachlor epoxide in the culture medium consisting of birch sawdust and rice bran, and found that $P$. acanthocystis completely eliminated heptachlor after 28 days of incubation. These observations suggest that Phlebia species like $P$. acanthocystis have strong activity to degrade and transform of organochlorine pesticides. Nevertheless, there is still a lack of information on metabolic and enzymatic mechanism for PCP degradation by Phlebia species and led us to pay attention to these fungi in their capability to degrade and transform PCP.

Therefore, the main objectives of the present study were: (1) to evaluate the tolerant and degradation ability of Phlebia species to PCP; (2) to detect and identify degradation products of PCP by $P$. acanthocystis; (3) to elucidate the involvement of enzymes such as ligninolytic enzymes and cytochrome P450 enzymes in PCP degradation; (4) to propose the possible degradation pathway of PCP by $P$. acanthocystis.

\section{Materials and methods \\ Fungus and culture conditions}

Stock cultures of Phlebia acanthocystis TMIC34875, Phlebia tremellosa TMIC30511 and Phlebia aurea TMIC33908, which have been obtained from Tottori Mycological Institute (Tottori, Japan), was maintained on potato dextrose agar (PDA, Difco, Detroit, MI, USA) slant tubes at $4{ }^{\circ} \mathrm{C}$. The fungi were transferred and incubated on 9-cm-diameter PDA plates at $30^{\circ} \mathrm{C}$ for $5-7$ days before being used for inoculum preparation. The mycelium mats on the PDA plates were transferred to a sterilized blender cup containing $50 \mathrm{~mL}$ of sterilized water and were homogenized for $30 \mathrm{~s}$. One milliliter of this homogenate was inoculated into a $100-\mathrm{mL}$ Erlenmeyer flask containing $10 \mathrm{~g} / \mathrm{L}$ glucose as a carbon source, $1.20 \mathrm{mM}$ ammonium tartrate as a nitrogen source, $20 \mathrm{mM}$ sodium acetate, and trace element solution, as low-nitrogen (LN) medium or potato dextrose broth (PDB, Difco, Detroit, MI, USA), after the initial $\mathrm{pH}$ of the medium was adjusted to 4.5 with $1 \mathrm{M} \mathrm{HCl}$. The cultures were pre-incubated statically at $30{ }^{\circ} \mathrm{C}$ in the dark under ambient atmospheric conditions.

\section{PCP tolerance experiments}

The tolerance experiments were carried out in $9-\mathrm{cm}$ diameter Petri dish plates containing PDA solid medium. PCP (Wako Pure Chemical Industries, purity 99\%) dissolved in acetone at different concentrations was prepared as stock solutions and added to sterilized PDA medium after autoclaving and cooling, and then the plate was placed in a Clean Bench for several hours to evaporate acetone before inoculation. Mycelium plugs (5 mm) was punched from 5-day-old fungal colony in agar plates and placed in the center of Petri plates and incubated at $30{ }^{\circ} \mathrm{C}$ in darkness. The colony diameter and mycelium dry weight were measured by daily to assert the tolerance of the strain to PCP, until the complete coverage of the plates. As a control, the plates without PCP were carried out according to the above method. To evaluate the ability of fungus to maintain growth activity in the presence of PCP, two tolerance coefficients were defined using the following equation [15]:

$$
\mathrm{TCE}=\mathrm{CDS} / \mathrm{CDC},
$$

where TCE is the tolerance coefficient in colony expansion, $\mathrm{CDS}$ and $\mathrm{CDC}$ are the colony diameter in the sample and control, respectively. 


$$
\mathrm{TCB}=\mathrm{DWS} / \mathrm{DWC},
$$

where TCB is the tolerance coefficient in mycelium biomass, DYS and DYC are the dry weight of mycelium in the sample and control, respectively.

\section{Degradation experiments}

Based on the results of tolerance test, $P$. acanthocystis was selected for the degradation experiment. After pre-incubation for 5 days, PCP dissolved in acetone was aseptically added to inoculated flask to make the final concentration of PCP in each sample was $25 \mu \mathrm{M}$. The flask was sealed with a glass stopper and sealing tape to prevent the volatilization of PCP, after the $100 \%$ oxygen was added to the headspace of each flask. The samples were placed statically for 15 days at $30{ }^{\circ} \mathrm{C}$ in dark conditions. As a control, the fungal cultures were killed by autoclaving $\left(121^{\circ} \mathrm{C}, 20 \mathrm{~min}\right)$ before the addition of the substrate. After incubation, the cultures were homogenized with $20 \mathrm{~mL}$ of acetone, and the biomass was removed by centrifugation at $3000 \mathrm{rpm}$ for $10 \mathrm{~min}$. The resulting supernatant was analyzed by high-performance liquid chromatography (HPLC) to quantify the recovery of substrate after filtration with a membrane filter $(0.45 \mu \mathrm{m})$. HPLC was performed on a Jasco PU-1580 intelligent pump and a Jasco MD-1510 multiwavelength detector fitted with an Inertsil ODS-3 column (150 mm) with an inner diameter of $4.6 \mathrm{~mm}$ (GL Science Inc., Tokyo, Japan). The supernatant was eluted with $80 \%$ acetonitrile in $0.1 \%$ trifluoroacetic acid aqueous solution at a flow rate of $1 \mathrm{~mL} / \mathrm{min}$.

For the identification of metabolites, a part of supernatant was evaporated to remove acetone, and was extracted three times with ethyl acetate. The residual biomass was air-dried and Soxhlet-extracted for $12 \mathrm{~h}$ with ethyl acetate. The ethyl acetates were mixed and dried with anhydrous sodium sulfate and then was concentrated to dryness under reduced pressure. The metabolite was analyzed by gas chromatography/mass spectrometry (GC/MS) performed on an HP 6890 GC system linked to an HP 5973 mass selective detector and a $30-\mathrm{m}$ fused DB-5MS column $(0.25 \mathrm{~mm}$ inside diameter, $0.25 \mu \mathrm{m}$ film thickness). The oven temperature was programmed at $80^{\circ} \mathrm{C}$ for $3 \mathrm{~min}$, followed by a linear increase to $300^{\circ} \mathrm{C}$ at $20^{\circ} \mathrm{C} \mathrm{min}{ }^{-1}$ and held for $3 \mathrm{~min}$. The injector temperature was set at $250^{\circ} \mathrm{C}$, and a $1-\mu \mathrm{L}$ splitless injection was used. Acetic anhydride/pyridine was used for acetyl derivatization analysis as described previously [12].

\section{Cytochrome P450 inhibitors experiments}

The inhibition studies of cytochrome P450 monooxygenase (Cyt P450) were conducted as previously described [15]. The culture of $P$. acanthocystis was incubated using $\mathrm{LN}$ medium for 5 days at $30{ }^{\circ} \mathrm{C}$ in the dark, and then the Cyt P450 inhibitor piperonyl butoxide (PB) or 1-aminobenzotriazole (ABT) was added to obtain final concentration of $1.0 \mathrm{mM}$. The flasks were agitated to homogenate the inhibitor solutions with the medium. After $120 \mathrm{~min}$, substrates were added to give a final concentration of $25 \mu \mathrm{M}$. Subsequently, fungal cultures were further incubated for 10 days. The analysis method of PCP and its degradation products is the same as the above description.

\section{Enzyme assays}

The extracellular ligninolytic enzymes activities were investigated by spectrophotometric assays. After filtration of supernatant of a previously centrifuged sample (10 min at $3000 \mathrm{rpm}$ ) from LN culture using a membrane $0.45-\mu \mathrm{m}$ filter to further remove the impurities, the enzyme activity of the crude supernatant was measured using a UV-Vis spectrophotometer at $20{ }^{\circ} \mathrm{C}$. Laccase (Lac) activity was assayed by monitoring the oxidation of 2,2-azinobis (3-ethylbenzothiazoline-6-sulfonic acid) in $20 \mathrm{mM}$ sodium acetate buffer ( $\mathrm{pH} 4.0)$ at $420 \mathrm{~nm}$ absorbance. Manganese peroxidase ( $\mathrm{MnP}$ ) activity was measured by monitoring the oxidation of $20 \mathrm{mM}$ 2,6-dimethoxyphenol at $470 \mathrm{~nm}$ in $50 \mathrm{mM}$ malonate buffer ( $\mathrm{pH} 4.5)$ in the presence of $20 \mathrm{mM} \mathrm{MnSO}_{4}$. Lignin peroxidase (LiP) activity was measured by monitoring the formation of veratryl aldehyde from veratryl alcohol in $20 \mathrm{mM}$ sodium tartrate buffer (pH 3.0) at $310 \mathrm{~nm}$. In the determination of $\mathrm{MnP}$ and LiP activity, $0.2 \mathrm{mM} \mathrm{H}_{2} \mathrm{O}_{2}$ was added to start the reaction. All the enzyme activities were expressed as international units $(U)$, where one unit is defined as the amount of enzyme that oxidizes $1 \mu \mathrm{mol}$ of substrate in $1 \mathrm{~min}$.

\section{In vitro degradation experiments}

The potential of extracellular enzymes to degrade and transform PCP was evaluated using the extracellular fluid obtained from $P$. acanthocystis cultures with LN medium. The extracellular fluid was collected and filtered by a membrane filter $(0.45 \mu \mathrm{m})$ after 10-days of incubation. PCP solution was added to $20 \mathrm{~mL}$ of extracellular fluid to obtain a final concentration of $25 \mu \mathrm{M}$, and then treated at $30{ }^{\circ} \mathrm{C}$. After $24 \mathrm{~h}$, PCP concentration and formation of metabolites were determined according to the above method.

\section{Mineralization experiments}

Labeled $\left[{ }^{14} \mathrm{C}\right] \mathrm{PCP}$ (purity $>98 \%$, from Sigma Chemical Co., St. Louis, MO, USA) was dissolved in ethanol (99\%) to obtain a stock solution with a specific activity of $16 \mathrm{kBq} / \mathrm{mL}$. The fungal cultivation with $P$. acanthocystis was carried out as described above using LN medium. 
After incubation for 5 days, $50 \mu \mathrm{L}$ of a $5 \mathrm{mM}\left[{ }^{14} \mathrm{C}\right] \mathrm{PCP}$ solution was added as a substrate to each inoculated flask. Incubation was carried out in a dark chamber at $30{ }^{\circ} \mathrm{C}$. As a control, the fungal cultures were sterilized by autoclaving $\left(121{ }^{\circ} \mathrm{C}, 20 \mathrm{~min}\right)$ to monitor abiotic PCP degradation, before the addition of the $\left[{ }^{14} \mathrm{C}\right] \mathrm{PCP}$. The trapping of ${ }^{14} \mathrm{CO}_{2}$ was conducted by method of Mori and Kondo [16]. The headspace of each flask was flushed with oxygen every 3 days, and ${ }^{14} \mathrm{CO}_{2}$ was trapped in $10 \mathrm{~mL}$ of an ethanolamine-containing scintillation fluid (Ultima gold EX:methanol:ethanolamine $=5: 4: 1)$. Trapped ${ }^{14} \mathrm{CO}_{2}$ was measured according to the ${ }^{14} \mathrm{C}$ radioactivity on a liquid scintillation counter Accuflex7400 (Aloka, Japan).

All experiments in this study were performed in triplicate. The results are reported as the average of triplicate determinations.

\section{Results and discussion Fungal tolerance to PCP}

Table 1 shows the results of fungal tolerance test in PDA medium with PCP of different concentrations. When these strains were cultured with low concentration of PCP $(2.0 \mathrm{mg} / \mathrm{L})$, the tolerance coefficients of three fungi were more than 0.9 after 8 days, except for TCB value of $P$. tremellosa, which indicated that these fungi had better tolerance to low concentration of PCP. On the other hand, low tolerance levels were found in fungal growth with high concentrations $(20 \mathrm{mg} / \mathrm{L})$ of PCP. For example, TCB value of $P$. acanthocystis was only 0.7 , while TCB values of $P$. tremellosa and $P$. aurea were lower than 0.5 , during the whole incubation period. TCE values also showed similar results to TCB values in these fungi. By comparison, $P$. acanthocystis was more tolerant for $\mathrm{PCP}$ than other two fungi. In addition, the tolerance coefficients of these strains to different concentrations of PCP increased with incubation time, which was attributed to the increased adaptability of the strains and the decreased concentration of PCP by fungal degradation.
As is well known, white rot fungi are the most tolerant microorganisms to a variety of high-concentration pollutants, which has unique biodegradation ability. Therefore, it is the first work to screen the degradation strains that can grow efficiently in the presence of high concentrations of PCP, in order to develop an effective biological resource that can be applied to the remediation of PCP-contaminated sites. Nevertheless, there is little information regarding the tolerance of white rot fungi to PCP. Alleman et al. [17] investigated the toxicity of PCP to six species of white rot fungi, and they found all species failed to grow at PCP concentrations higher than $5 \mathrm{mg} / \mathrm{L}$, when added as a 1-day-old fungal colony. In our experiment, 5-day-old fungal colonies were used and showed better tolerance to PCP, indicating that growth of the fungus, resulting in greater biomass, may favorably affect tolerance to PCP. It is believed that the presence of organochlorine insecticides induces the production of protective proteins, which counteracts the adverse effects of insecticides on the fungal growth [18]. Actually, the concentration of PCP in the actual environment is much lower than that of PCP used in this experiment, thus it is considered that these Phlebia species with better tolerance might be valuable as a useful resource for the biodegradation and remediation of PCP pollution.

\section{Biodegradation of PCP by P. acanthocystis}

Figure 1 shows the recovery concentration of PCP in LN and PDB liquid media after degradation of P. acanthocystis. The concentration of PCP in control cultures during the whole incubation period was higher than $97 \%$ of the initial concentration, which shows that the abiotic degradation such as volatilization and photolysis was not significant in the experiments. Approximately $64 \%$ of the initial amount PCP was eliminated at 4 days, and almost all PCP has disappeared after 8 days in LN medium. In contrast, the fungal degradation curve of $\mathrm{PCP}$ in $\mathrm{PDB}$ medium is different from that in LN medium, $24.3 \%$ of PCP still remained in fungal cultures, even at the end of

Table 1 The tolerance coefficient of white rot fungi to different concentrations of PCP in PDA medium

\begin{tabular}{|c|c|c|c|c|c|c|c|c|c|}
\hline \multirow[t]{2}{*}{ Strains } & \multirow[t]{2}{*}{$\mathrm{PCP}(\mathrm{mg} / \mathrm{L})$} & \multicolumn{4}{|l|}{ TCE } & \multicolumn{4}{|l|}{ TCB } \\
\hline & & $2 d$ & $4 d$ & $6 \mathrm{~d}$ & $8 d$ & $2 d$ & $4 d$ & $6 d$ & $8 d$ \\
\hline \multirow[t]{2}{*}{ Phlebia acanthocystis } & 2.0 & 0.91 & 0.90 & 0.93 & 0.95 & 0.89 & 0.88 & 0.94 & 0.96 \\
\hline & 20.0 & 0.72 & 0.75 & 0.78 & 0.80 & 0.66 & 0.69 & 0.70 & 0.73 \\
\hline \multirow[t]{2}{*}{ Phlebia tremellosa } & 2.0 & 0.84 & 0.85 & 0.87 & 0.90 & 0.73 & 0.74 & 0.77 & 0.83 \\
\hline & 20.0 & 0.51 & 0.52 & 0.56 & 0.60 & 0.33 & 0.32 & 0.37 & 0.41 \\
\hline \multirow[t]{2}{*}{ Phlebia aurea } & 2.0 & 0.87 & 0.90 & 0.91 & 0.93 & 0.80 & 0.84 & 0.85 & 0.90 \\
\hline & 20.0 & 0.54 & 0.59 & 0.60 & 0.66 & 0.38 & 0.42 & 0.44 & 0.50 \\
\hline
\end{tabular}



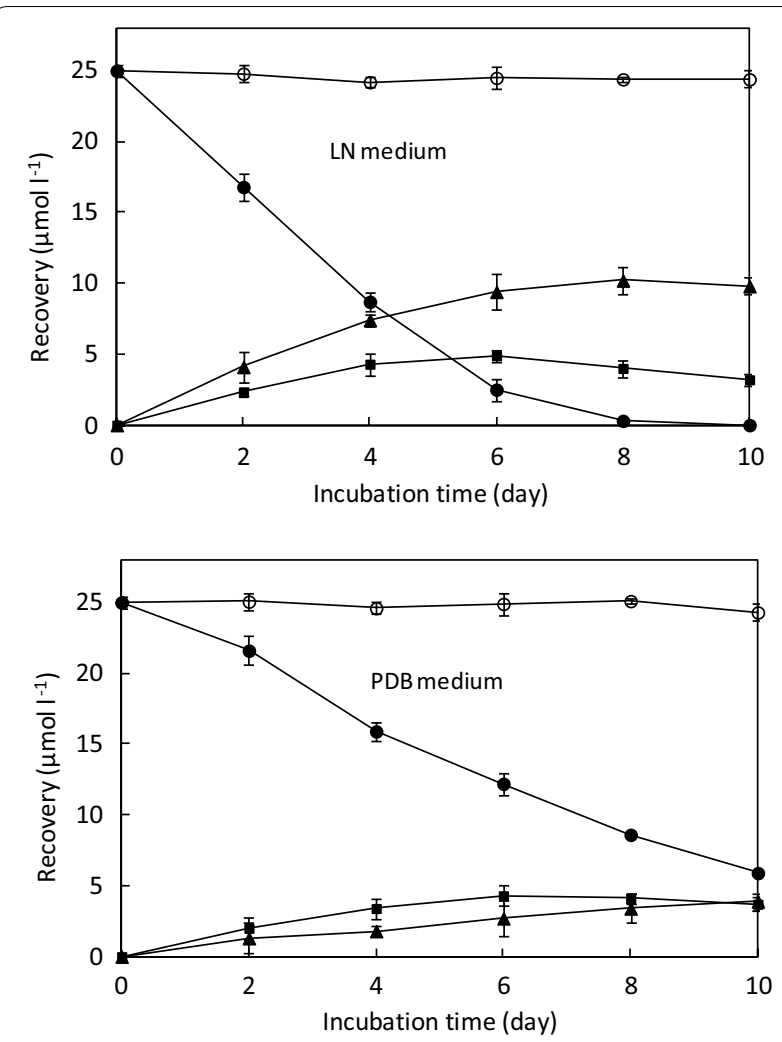

Fig. 1 Time course for degradation of PCP (closed circles) and formation of PCA (closed triangles) and TCHQ (closed squares) by P. acanthocystis in LN and PDB media. Recovery of PCP from control cultures was also shown (open circles). Values are mean \pm SD of triplicate samples

incubation. Generally, the ligninolytic activity of some wood rot fungi is stimulated by nitrogen limitation, and is inhibited under high-nitrogen conditions [19]. P. acanthocystis, used as a representative wood rot fungus, obviously degraded PCP in low-nitrogen culture. Therefore, the results obtained in this study confirmed that $P$. acanthocystis had higher ability to degrade PCP under lownitrogen conditions, and suggested that degradation of PCP might be related to the ligninolytic enzyme system.

\section{Detection and identification of PCP metabolites}

From the second day of the degradation experiment of PCP, a metabolite was detected in the extracts of fungal culture, and the retention time and mass spectrum of this metabolite identical to authentic pentachloroanisole (PCA, Table 2). Accumulation of PCA was observed more in LN medium than in PDB medium during whole incubation periods. After 10 days of incubation, about $9.8 \mu \mathrm{M}$ of PCA was detected from LN medium with $P$. acanthocystis, while only $3.9 \mu \mathrm{M}$ of PCA was produced in PDB medium (Fig. 1). Additionally, another metabolite was observed in acetyl derivatized culture extracts, and the mass spectrum of this compound showing ion peaks at $m / z 330[\mathrm{M}](\mathrm{Cl}=35), 288\left[\mathrm{M}-\mathrm{COCH}_{2}\right]^{+}$and $246\left[288-\mathrm{COCH}_{2}\right]^{+}$, is almost identical to that of acetylation products of $p$-tetrachlorohydroquinone (TCHQ, Table 2). As shown in Fig. 1, the formation of TCHQ in both media showed almost same level, which ranged from 3.2 to $3.7 \mu \mathrm{M}$ after 10 days of incubation (Fig. 1). In this study, the formation of PCA and TCHQ indicated that $P$. acanthocystis initially transform PCP via methylation and oxidation, respectively. An interesting result was found that during the whole incubation period, formation of PCA was greater than that of TCHQ in LN medium, while formation of PCA was lower than that of TCHQ in PDB medium. In this study, PCP was more easily methylated to $\mathrm{PCA}$ in low-N conditions, suggesting that the methylation reaction of PCP is likely to be related to fungal ligninolytic enzymes. To determine the localization of PCP-degrading enzyme, the distribution of PCA in extracellular filtrate and mycelium was determined after 10 days, and it has to be emphasized that PCA is only detected in the extract of extracellular filtrate, but almost not in the extract of mycelium (data not shown), which further indicates that the methylation of PCP to produce PCA is catalyzed by extracellular enzymes.

\section{Biotransformation of PCA and TCHQ by P. acanthocystis}

The formation of PCA and TCHQ, however, did not account for the total loss of PCP in the culture,

Table 2 Mass spectra of the metabolites of PCP from culture of $P$. acanthocystis

\begin{tabular}{|c|c|c|}
\hline Metabolites or derivatized metabolites & Formula & Mass spectra $m / z$ (relative intensity) \\
\hline PCA & $\mathrm{C}_{7} \mathrm{H}_{3} \mathrm{OCl} I_{5}$ & $\begin{array}{l}284(25.1), 282(70.3), 280(100), 278(63.3), 269(23.2), 267(65.7), 265(93.7), 263(60.6), 241 \\
\quad(18.2), 239(51.5), 237(74.7), 235(47.5), 212(5.1), 200(4.0), 177(7.1), 163(29.6)\end{array}$ \\
\hline $\begin{array}{l}\text { Tetrachloro-1,4-diacetoxybenzene (acety- } \\
\text { lation products of TCHQ) }\end{array}$ & $\mathrm{C}_{10} \mathrm{H}_{6} \mathrm{O}_{4} \mathrm{Cl}_{4}$ & $\begin{array}{l}334(3.9), 332(6.5), 330(4.9), 292(6.4), 290(10.9), 288(9.4), 252(11.2), 250(50.2), 248(100), 246 \\
\quad(80.2)\end{array}$ \\
\hline $\begin{array}{l}\text { Tetrachloro-1-acetoxy-4-methoxyben- } \\
\text { zene (acetylation products of TCMP) }\end{array}$ & $\mathrm{C}_{9} \mathrm{H}_{6} \mathrm{O}_{3} \mathrm{Cl}_{4}$ & $\begin{array}{l}306(3.9), 304(5.5), 302(4.8), 266(12.0), 264(50.3), 262(100), 260 \text { (76.8), } 251 \text { (5.9), } 249(28.4), 247 \\
\quad(56.8), 245(44.0)\end{array}$ \\
\hline TCDB & $\mathrm{C}_{8} \mathrm{H}_{6} \mathrm{O}_{2} \mathrm{Cl}_{4}$ & $\begin{array}{l}280 \text { (10.8), } 278(31.6), 276(68.5), 274(42.6), 265 \text { (13.4), } 263 \text { (56.1), } 261 \text { (100), } 259(83.1), 213 \\
\quad(17.6), 211(49.0), 209(50.9)\end{array}$ \\
\hline
\end{tabular}




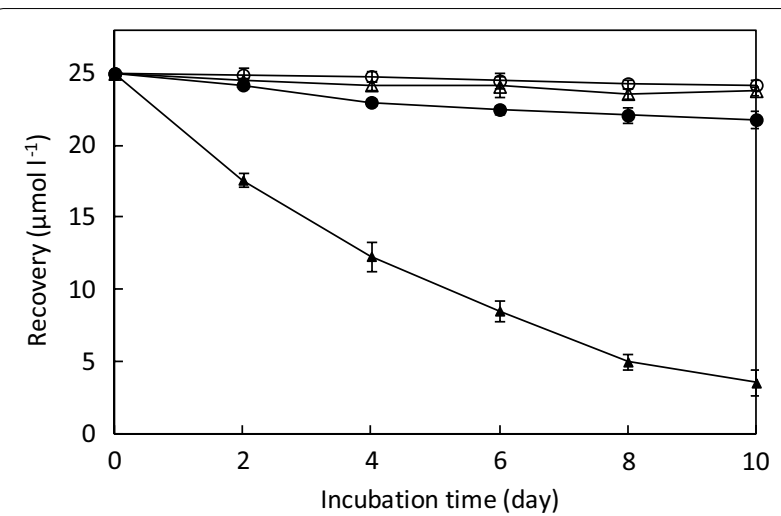

Fig. 2 Time courses for degradation of PCA (closed circles) and TCHQ (closed triangles) by P. acanthocystis in LN medium. Recovery of PCA (open circles) and TCHQ (open triangles) from control cultures was also shown. Values are mean \pm SD of triplicate samples

suggesting that methylation and oxidation are only the first step in the transformation of PCP, and PCA or/and TCHQ may be further converted. Thus the degradation of PCA and TCHQ $(25 \mu \mathrm{M})$ were investigated in LN medium using $P$. acanthocystis. As a result, about $85 \%$ of TCHQ disappeared in fungal culture incubated for 10 days, when TCHQ was used as a degradation substrate (Fig. 2).

A new metabolite appears in the extracts of fungal culture after acetyl derivatization by GC/MS analysis, and main ion peaks in its mass spectrum are shown in Table 2. The heaviest ion peak at $\mathrm{m} / z 302$ containing the isotopic cluster of four chlorine atoms was observed in the mass spectrum of acetylated metabolite. The difference of $m / z 42$ between ion peaks at $m / z$ 302 and $m / z 260$ is very typical for mass spectra of acetylated compound, representing a loss of $\left[\mathrm{COCH}_{2}\right]^{+}$, whereas difference of $\mathrm{m} / z 15$ between ion peaks at $\mathrm{m} / \mathrm{z}$ 260 and $m / z 245$ represents the loss of methyl group $\left[\mathrm{CH}_{3}\right]^{+}$. Mass spectral analysis suggests the presence of a hydroxyl group and a methyl group on this metabolite molecule and was identified as tetrachloro-4-methoxyphenol (TCMP). From the underivatized extracts of fungal culture, another metabolite was detected by GC/MS and its mass spectrum showed a molecular ion peak at $m / z 274$ which has the characteristic of four chlorine atoms, and fragment ions at $\mathrm{m} / z 259$ and $\mathrm{m} / z$ 209 (Table 2). The loss of a $\left[\mathrm{CH}_{3}\right]^{+}$from ion peak of $\mathrm{m} / \mathrm{z} 274$ gives rise to a fragment ion of $\mathrm{m} / \mathrm{z} 259$ with four chlorine atoms. Ion peak at $m / z 209$ has the isotopic cluster of three chlorine atoms, which represent the loss of $\left[\mathrm{CH}_{3}\right]^{+}$and a chlorine atom from ion peak of $m / z 259$. The information obtained from above analysis suggests the presence of four chlorine atoms and two methyl groups in the metabolite molecule, and this metabolite was identified as tetrachloro-1,4-dimethoxybenzene (TCDB).

The detection of TCMP and TCDB indicates that methylation is important initial reaction in transformation of TCHQ by P. acanthocystis. It is worth mentioning that several additional metabolites from TCHQ were also found in fungal cultures by GC/MS analysis, nevertheless it is not possible to determine the structures due to their low concentration. When PCA was added as a degradation substrate, the decrease rate of PCA concentration was extremely slow, and over 90\% PCA was recovered from the fungal culture until the end of incubation (Fig. 2). As can be seen from these results, it is difficult for $P$. acanthocystis to transform PCA in LN medium, that is to say, PCA should appear to be a dead-end product in the transformation of PCP by $P$. acanthocystis. None of the above metabolites were found in the control cultures.

\section{Degradation pathway of PCP by P. acanthocystis}

Based on the identification of the metabolites detected from this study, the proposed degradation pathway of PCP in P. acanthocystis is presented in Fig. 3. These data clearly indicate two branch pathways from PCP in P. acanthocystis: (1) first methylated to produce PCA, which may be a dead-end in the metabolic pathways; and (2) first oxidized to produce TCHQ, which can further undergo successive methylation to produce TCMP and TCDB. It seems more likely that the former is the secondary route, and latter is the major route, because TCHQ is converted more easily than PCA, after they are produced.

At present, the fungal groups most commonly used in research of PCP transformation are basidiomycetes, agents of white rot. Among these, the genus

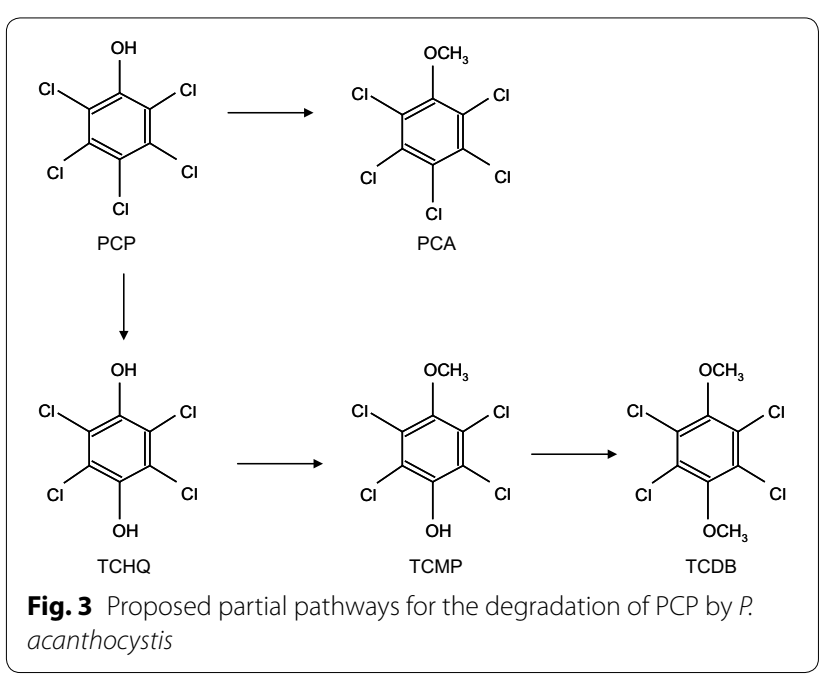


Phanerochaete, Anthracophyllum and Trametes have received more attention. However, little is actually known about the metabolic pathway of PCP in white rot fungi. Bosso and Cristinzio [20] showed also that the main reaction of PCP degradation in A. discolor was methylation with the formation of $\mathrm{PCA}$, and the second reaction was hydroxylation in the form of TCHQ, which is then methylated to generate TCDB, followed by successive reactions to form dechlorination products. A complete dechlorination pathway of PCP in P. chrysosporium different from that in our study has been reported by Reddy and Gold [21], who found that PCP is first oxidized by LiP or $\mathrm{MnP}$ to tetrachlorobenzoquinone, which apparently is further degraded by two parallel pathways with several cross-pathway steps. A comparison between our results and those of the paper describing the fungal transformation of PCP suggested that the initial metabolic pathway of PCP in P. acanthocystis seems to be similar to that in $A$. discolor, though the complete pathway of PCP in our study is still unclear.

\section{Ligninolytic enzymes activities}

In order to determine whether ligninolytic enzymes are involved in PCP degradation, the activities of three ligninolytic enzymes were evaluated in extracellular fluid from $P$. acanthocystis culture in the absence and presence of PCP during the incubation period. As can be seen from Table 3, the activity of LiP is the highest among the three enzymes, which is not surprising because it has been widely reported that white rot fungi produces LiP in low-nitrogen condition. The activities of LiP and $\mathrm{MnP}$ increased with time, whereas the Lac activity was only detected at trace level within 10 days, even in the $100 \mu \mathrm{M}$ PCP-added sample. The low quantity of Lac produced in this study is similar with other studies, where it is indicated that white rot fungi produce constitutively low concentrations of Lac. It has been reported that the higher phenol concentrations pronounced the loss of Lac activity [22]. In general, the increase of LiP activity was found in extracellular fluid of fungal culture with addition of PCP, especially in a high concentration $(100 \mu \mathrm{M})$ of PCP. The maximum LiP activities of the values 137.4 $\mathrm{U} / \mathrm{L}$ and $169.6 \mathrm{U} / \mathrm{L}$ were obtained from the samples with addition of $25 \mu \mathrm{M}$ and $100 \mu \mathrm{M}$ PCP, respectively, on the sixth day of incubation; afterwards the activity slowly decreased. The maximal LiP activity was reached after 6 days of incubation and was also higher PCP degradation efficiency and accumulation of PCA and TCHQ (Fig. 1). By comparison, the activity of $\mathrm{MnP}$ was only slightly increased in the extracellular fluid from $100 \mu \mathrm{M}$ PCP-added sample, with a maximum of $73.4 \mathrm{U} / \mathrm{L}$ on the tenth day of incubation.

The aggressive wood rot fungi which are widely spread in the world, thanks to their enzymes it could be an excellent candidate for direct biodegradation of pollutants. Most of the current research focused mostly on the activities of these ligninolytic enzymes to understand the enzymatic mechanism of PCP degradation in wood rot fungi, and lignolytic enzymes LiP, MnP and Lac were found to play important role in organic pollutant degradation [7-11]. Usually, when fungi increase their enzymatic activity, they showed a higher degradation of PCP. In the present study, activities of ligninolytic enzymes were measured during degradation of $\mathrm{PCP}$ by $P$. acanthocystis. The activities of LiP and MnP were observed, and only LiP presented positive correlation with PCP removal. It was noted from our results that LiP activity was promoted by the addition of PCP, therefore it was presumed that $P$. acanthocystis able to degrade PCP by ligninolytic enzymes system at least in part, as described above for this fungus showed higher PCP degradation under low-nitrogen condition than that under

Table 3 Enzyme activities produced by P. acanthocystis during the incubation with or without addition of PCP

\begin{tabular}{|c|c|c|c|c|c|c|}
\hline \multirow[t]{2}{*}{ Enzymes } & \multirow[t]{2}{*}{ PCP $(\mu \mathrm{M})$} & \multicolumn{5}{|c|}{ Activities (U/L) } \\
\hline & & $2 d$ & $4 d$ & $6 \mathrm{~d}$ & $8 d$ & $10 \mathrm{~d}$ \\
\hline \multirow[t]{3}{*}{ LiP } & 0 & $87.3 \pm 2.5$ & $104.3 \pm 12.3$ & $103.2 \pm 14.6$ & $126.3 \pm 5.9$ & $119.8 \pm 11.5$ \\
\hline & 25 & $97.3 \pm 14.1$ & $98.3 \pm 9.8$ & $137.4 \pm 17.6$ & $134.2 \pm 8.0$ & $134 \pm 14.7$ \\
\hline & 100 & $89.6 \pm 15.6$ & $134.6 \pm 20.3$ & $169.6 \pm 5.7$ & $149.3 \pm 17.3$ & $145 \pm 9.7$ \\
\hline \multirow[t]{3}{*}{$\mathrm{MnP}$} & 0 & $47.7 \pm 6.8$ & $59.6 \pm 9.5$ & $61.3 \pm 7.3$ & $58.9 \pm 3.5$ & $56.3 \pm 7.9$ \\
\hline & 25 & $54.5 \pm 4.6$ & $50.6 \pm 6.0$ & $63.9 \pm 9.4$ & $70.8 \pm 5.5$ & $61.5 \pm 14.5$ \\
\hline & 100 & $51.6 \pm 11.2$ & $62.2 \pm 13.8$ & $65.8 \pm 2.2$ & $61.8 \pm 6.0$ & $73.4 \pm 12.8$ \\
\hline \multirow[t]{3}{*}{ Lac } & 0 & $3.2 \pm 0.3$ & $2.6 \pm 0.2$ & $1.9 \pm 0.1$ & $2.1 \pm 1.6$ & $0.6 \pm 0.6$ \\
\hline & 25 & $1.2 \pm 0.2$ & $3.0 \pm 1.9$ & $2.0 \pm 0.8$ & $3.6 \pm 0.2$ & $2.6 \pm 1.3$ \\
\hline & 100 & $1.9 \pm 0.7$ & $2.3 \pm 0.6$ & $3.7 \pm 1.2$ & $3.1 \pm 0.9$ & $2.9 \pm 1.1$ \\
\hline
\end{tabular}

Values are mean \pm SD of triplicate samples, $d$ days 
high-nitrogen condition. In fact, the degradation of these compounds may be attributed to the action of other extracellular fungal enzymes such as phenoloxidases and cellobiase dehydrogenases, which may participate concomitantly with ligninolytic enzymes in the degradation process [22].

\section{In vitro degradation of PCP by extracellular fluid}

The in vitro experiment for degradation of PCP, PCA and TCHQ using extracellular fluid from $P$. acanthocystis was investigated. As shown in Table 4, 88.4\% and $82.6 \%$ of PCP were recovered from PCP-added extracellular fluid after $12 \mathrm{~h}$ and $24 \mathrm{~h}$, respectively. PCA and trace TCHQ were detected from extracellular fluid by GC/MS when PCP was used as degradation substrate; whereas when PCA and TCHQ were added to extracellular fluid as substrates, they were recovered by $93.2 \%$ and $79.6 \%$ after $24 \mathrm{~h}$, respectively. As degradation products, TCMP was found from extracellular fluid with addition of TCHQ, but no degradation products of PCA were obtained. The results showed that the extracellular enzyme systems such as LiP was responsible for the transformation of PCP to PCA and TCHQ, and the transformation of TCHQ to TCMP, which also verified the previous hypothesis that extracellular enzymes participated in the degradation of PCP. Reddy and Gold [21] reported the degradation of PCP by $P$. chrysosporium, and believed that the multi-step pathway is initiated by a LiP- or MnPcatalyzed oxidative dechlorination reaction. Zupan et al.
[23] have also reported that PCP degradation by white rot fungus Dichomitus squalens is related to the production of extracellular ligninolytic enzymes such as LiP and MnP.

\section{Effects of Cyt P450 inhibitors}

The effects of the Cyt P450 inhibitors on the degradation of PCP and TCHQ by P. acanthocystis for 10 days were investigated. The most noticeable difference was found in the degradation of PCP between the uninhibited and inhibited samples, and lower degradation activity was observed in cultures with 1.0 mM BP and ABT (Table 5). After 10 days of incubation, degradation rates of PCP decreased by more than $60 \%$, when both Cyt P450 inhibitors were added. As a degradation product of $\mathrm{PCP}$, almost no significant decrease of PCA formation was observed after addition of Cyt P450 inhibitors, however, the accumulation of TCHQ clearly decreased from 3.9 to 0.4 and $0 \mu \mathrm{M}$ in $\mathrm{PB}$ and $\mathrm{ABT}$-treated samples, respectively. Moreover, TCMP and TCDB were found to disappear completely in Cyt $\mathrm{P} 450$ inhibition conditions. The effects of $\mathrm{PB}$ and $\mathrm{ABT}$ in the inhibition test strongly suggesting that Cyt P450 are involved in initial oxidation of PCP to TCHQ, but seems not be involved in the methylation of PCP to PCA.

On the other hand, when TCHQ was treated as substrate, there was no significant change in their degradation rates after addition of inhibitors. As shown in Table 5, the degradation rates of TCHQ in the PB and

Table 4 In vitro degradation of PCP by extracellular fluid from P. acanthocystis

\begin{tabular}{|c|c|c|c|c|c|c|}
\hline \multirow[t]{2}{*}{ Substrates } & \multicolumn{2}{|c|}{ Recovery concentration $(\mu \mathrm{M})$} & \multicolumn{4}{|c|}{ Recovery of metabolites } \\
\hline & $12 \mathrm{~h}$ & $24 \mathrm{~h}$ & $\overline{P C A}$ & TCHQ & TCMP & TCDP \\
\hline PCP & $22.5 \pm 1.6(90 \%)$ & $21.2 \pm 1.5(84.8 \%)$ & + & + & - & - \\
\hline PCA & $24.1 \pm 0.4(96.4 \%)$ & $23.6 \pm 1.1(94.4 \%)$ & & - & - & - \\
\hline $\mathrm{TCHQ}$ & $22.2 \pm 0.6(88.8 \%)$ & $19.7 \pm 1.2(78.8 \%)$ & - & & + & - \\
\hline
\end{tabular}

The initial concentration of all substrates was $25 \mu \mathrm{M}$. Values are mean \pm SD of triplicate samples. + detectable; - undetectable

Table 5 Effect of Cyt P450 inhibitors on PCP and TCHQ degradation in LN medium by P. acanthocystis at 10 days

\begin{tabular}{|c|c|c|c|c|c|c|}
\hline \multirow[t]{2}{*}{ Substrates } & \multirow[t]{2}{*}{ Treatments } & \multirow[t]{2}{*}{ Degradation rate (\%) } & \multicolumn{4}{|c|}{ Recovery of metabolites $(\mu \mathrm{M})$} \\
\hline & & & PCA & TCHQ & TCMP & TCDP \\
\hline \multirow[t]{3}{*}{ PCP } & Without inhibitor & 100 & 10.1 & 3.9 & + & + \\
\hline & $1.0 \mathrm{mM}$ PB & 40.3 & 9.5 & 0.4 & - & - \\
\hline & $1.0 \mathrm{mM} \mathrm{ABT}$ & 34.9 & 9.1 & - & - & - \\
\hline \multirow[t]{3}{*}{$\mathrm{TCHQ}$} & Without inhibitor & 86.9 & - & & + & + \\
\hline & $1.0 \mathrm{mM}$ PB & 81.3 & - & & + & + \\
\hline & $1.0 \mathrm{mM} \mathrm{ABT}$ & 77.5 & - & & + & + \\
\hline
\end{tabular}

The initial concentration of all substrates was $25 \mu \mathrm{M}$. + detectable; - undetectable 
ABT-treated cultures decreased by only $5.6 \%$ and $9.4 \%$ compared with those without inhibitors, respectively. Similarly, no effect on the accumulation of metabolites for the degradation of TCHQ was observed after addition of $\mathrm{PB}$ or $\mathrm{ABT}$. These results further confirmed that the formation of methylation products such as PCA, TCMP and TCDB is not mainly catalyzed by Cyt P450. There was no effect on fungal growth due to toxicity at the concentration of Cyt P450 inhibitors used in this experiment.

A number of reports have demonstrated that Cyt P450 from wood rot fungi belonging to genus Phlebia are capable of catalyzing oxygenation reactions of wide variety of exogenous compounds. However, no reports have been found in the literature concerning Cyt P450 involved in the degradation of PCP by Phlebia species. Ning and Wang [24] reported for the first time that the PCP oxidation was induced Cyt P450 in P. chrysosporium, and that PB significantly inhibited PCP transformation and TCHQ formation in this fungal culture. In our previous studies, Cyt P450 from Phlebia species including P. acanthocystis has been shown to be involved the initial hydroxylation of endrin [15]. The present study showed the significant inhibition of PCP degradation due to the addition of inhibitors, and the formation of TCHQ from PCP likely associated with Cyt P450. Therefore, it is assumed that in P. acanthocystis, most of PCP is catalyzed by Cyt P450 to form TCHQ via oxidation reaction and is then converted into TCMP and TCDB through continuous methylation reaction by other enzyme systems such as ligninolytic enzymes, which also presumably catalyzes the methylation of some PCP to PAC.

\section{Mineralization of $\left[{ }^{14} \mathrm{C}\right] \mathrm{PCP}$ by $P$. acanthocystis}

In the sterilized control, the mineralization rate was only less than $0.3 \%$ of the total $\left[{ }^{14} \mathrm{C}\right] \mathrm{PCP}$ during the experiment period (Fig. 4). Mineralization of $\left[{ }^{14} \mathrm{C}\right] \mathrm{PCP}$ began 6 days after incubation with a mineralization rate of $0.8 \%$. Subsequently, the mineralization rate increased rapidly, and the maximum mineralization rate was approximately $0.6 \%$ of the total $\left[{ }^{14} \mathrm{C}\right] \mathrm{PCP}$ per day during $12-15$ days. After 21 days of incubation, the trend of $\left[{ }^{14} \mathrm{C}\right] \mathrm{PCP}$ mineralization curve by $P$. acanthocystis increase has slowed down, which may be related to the lack of nutrients due to fungal consumption. Finally, $9.3 \%$ of $\left[{ }^{14} \mathrm{C}\right] \mathrm{PCP}$ was mineralized to ${ }^{14} \mathrm{CO}_{2}$ by $P$. acanthocystis incubated for 42 days. A novel result was found that the rates of ${ }^{14} \mathrm{CO}_{2}$ evolution could be increased slightly if additional glucose $(10 \mathrm{~g} / \mathrm{L})$ was added to the cultures at 24 days. After 18 days of glucose addition, the evolution of ${ }^{14} \mathrm{CO}_{2}$ increased by $0.8 \%$ compared with the treatment without glucose supplementation. Therefore, there is considered that fungal mineralization of $\left[{ }^{14} \mathrm{C}\right] \mathrm{PCP}$ was influenced by carbon sources concentration in the culture medium.

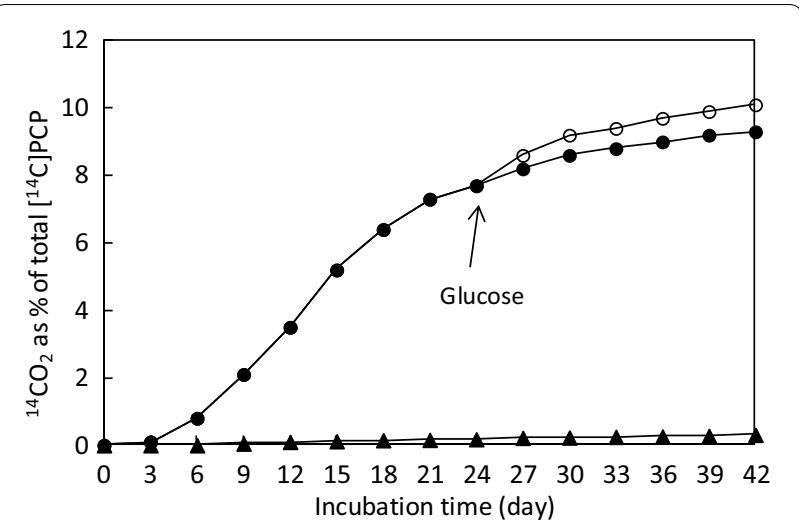

Fig. 4 Release of ${ }^{14} \mathrm{CO}_{2}$ from $\left[{ }^{14} \mathrm{C}\right] \mathrm{PCP}$ in P. acanthocystis culture without glucose supplement (closed circles) and with glucose supplement (open circles) for 42 days. Release of ${ }^{14} \mathrm{CO}_{2}$ from the sterilized cultures (closed triangles) is also shown. Values are mean \pm SD of triplicate samples. The arrow represents addition of $10 \mathrm{~g} / \mathrm{L}$ glucose at 24 days

Actually, the growth of wood rot fungi is usually inhibited when introduced into natural environments due to the low amount of available nutrients and competitive indigenous microorganisms. Therefore, the addition of available nutrients such as carbon sources is necessary to maintain the growth and degradation activity of wood rot fungi.

Some reports have also described wood rot fungi that are capable of mineralizing PCP in the pure culture or contaminated soil and may be useful for bioremediation of PCP-contaminated environments. Walter et al. [7] found that New Zealand white rot fungi T. versicolor converted roughly $20 \%$ of PCP to $\mathrm{CO}_{2}$ in liquid culture during a 30-day incubation period, and the mineralization rate was higher for $T$. versicolor compared to $P$. chrysosporium. Tuomela et al. [25] also reported that $T$. versicolor during 42 days of incubation mineralized about $29 \%$ of the PCP in autoclaved soil supplemented with straw. Several tropical basidiomycetes, Agrocybe perfecta, Trametes villosa, Psilocybe castanella and Peniophora cinerea were tested for their ability to mineralize PCP and the extent of PCP mineralization was between $3.07 \%$ and $8.15 \%$ for 120 days [26]. In comparison, the ability of $P$. acanthocystis to mineralize PCP in liquid culture is lower than of T. versicolor, but higher than of tropical basidiomycetes.

\section{Conclusion}

In the present study, 3 Phlebia species of wood rot fungi were used for determining the tolerance against PCP, and P. acanthocystis with best tolerance to PCP was selected for evaluating the ability to degrade and transform PCP. Results have demonstrated that $P$. acanthocystis can 
degrade PCP efficiently in low-nitrogen conditions and convert it into PCA and TCHQ in parallel, which are then further transformed into TCMP and TCDB. Involvement of ligninolytic enzymes and Cyt P450 in the fungal degradation of PCP has been confirmed by enzyme activity analysis, in vitro degradation test and Cyt P450 inhibitors test, and this finding can enhance the understanding of the role of this fungus towards enzyme-mediated degradation of PCP. The results obtained in the present study are important for the development of bioremediation methods because of the possibilities of using wood rot fungi for the decontamination and detoxification of PCPpolluted environments. Therefore, additional research is needed for evaluation of the effectiveness of degrading fungi in bioremediation of actual polluted environments, and optimization of the conditions for application.

\begin{abstract}
Abbreviations
PCP: pentachlorophenol; PCA: pentachloroanisole; TCHQ: $p$-tetrachlorohydroquinone; TCMP: tetrachloro-4-methoxyphenol;TCDB: tetrachloro-1,4-dimethoxybenzene; LN: low-nitrogen; PDA: potato dextrose agar; PDB: potato dextrose broth; TCE: tolerance coefficient in colony expansion; TCB: tolerance coefficient in mycelium biomass; LiP: lignin peroxidase; MnP: manganese peroxidase; Lac: laccase; Cyt P450: cytochrome P450 monooxygenase; PB: piperonyl butoxide; ABT: 1-aminobenzotriazole.
\end{abstract}

\section{Acknowledgements}

Not applicable.

\section{Authors' contributions}

PX performed the experiments, analyzed the data, and was a major contributor in writing the manuscript. All the authors have contributed to the manuscript and take all responsibilities for the entire content of the manuscript. All authors read and approved the final manuscript.

\section{Funding}

This work was supported by the Fundamental Research Funds for the Central Universities (No. 2572017CA08), the Heilongjiang Provincial Natural Science Foundation of China (No. LH2019D002), and the National Natural Science Foundation of China (No. 41201307).

\section{Availability of data and materials}

The datasets used and/or analyzed during the current study are available from the corresponding author on reasonable request.

\section{Competing interests}

The authors declare that they have no competing interests.

\section{Author details}

${ }^{1}$ College of Forestry, Northeast Forestry University, Hexing Road 26, Harbin 150040, China. ${ }^{2}$ Faculty of Agriculture, Kyushu University, Fukuoka 812-8581, Japan.

Received: 27 August 2019 Accepted: 8 January 2020

Published online: 16 January 2020

\section{References}

1. Cui Y, Liang L, Zhong Q, He Q, Shan X, Chen K, Huang F (2017) Theassociation of cancer risks with pentachlorophenol exposure: focusing on community population in the areas along certain section of Yangtze River in China. Environ Pollut 224:729-738
2. Zheng L, Liu Z, Yan Z, Zhang Y, Yi X, Zhang J, Zheng X, Zhou J, Zhu Y (2017) pH-dependent ecological risk assessment of pentachlorophenol in Taihu lake and Liaohe river. Ecotoxicol Environ Saf 135:216-224

3. Li X, Lin Z, Luo C, Bai J, Sun Y, Li Y (2015) Enhanced microbial degradation of pentachlorophenol from soil in the presence of earthworms: evidence of functional bacteria using DNA stable isotope probing. Soil Biol Biochem 81:168-177

4. Mendoza-Cantú A, Albores A, Fernández-Linares L, Rodriguez-Vázquez $R$ (2015) Pentachlorophenol biodegradation and detoxification by the white-rot fungus Phanerochaete chrysosporium. Environ Toxicol 15(2):107-113

5. Lamar RT, Davis MW, Dietrich DM, Glaser JA (1994) Treatment of a pentachlorophenol-and creosote-contaminated soil using the lignindegrading fungus Phanerochaete sordida: a field demonstration. Soil Biol Biochem 26(12):1603-1611

6. Boyle D (2006) Effects of $\mathrm{pH}$ cyclodextrins on pentachlorophenol degradation (mineralization) by white-rot fungi. J Environ Manag 80(4):380-386

7. Walter M, Boul L, Chong R, Ford C (2004) Growth substrate selection and biodegradation of PCP by New Zealand white-rot fungi. J Environ Manag 71(4):361-369

8. Okeke BC, Paterson A, Smith JE, WatsonCraik IA (1997) Comparative biotransformation of pentachlorophenol in soils by solid substrate cultures of Lentinula edodes. Appl Microbiol Biotechnol 48:563-569

9. Rubilar O, Feijoo G, Diez C, Lu-Chan TA, Moreira MT, Lema JM (2007) Biodegradation of Pentachlorophenol in Soil Slurry Cultures by Bjerkandera adusta and Anthracophyllum discolor. Ind Eng Chem Res 46:6744-6751

10. Bustamante M, Rubilar O, Diez MC (2014) Effect of soya lecithin on solubilization and biodegradation of pentachlorophenol by Anthracophyllum discolor. Am J Anal Chem 5:28-38

11. Ramesh C, Pattar MG (2009) Biodegradation of pentachlorophenol by white rot fungi isolated from forests of Western Ghats of Karnataka India. Curr Trends Biotech Pharm 3:417-427

12. Xiao P, Mori T, Kamei I, Kondo R (2011) Metabolism of organochlorine pesticide heptachlor and its metabolite heptachlor epoxide by white rot fungi, belonging to genus Phlebia. FEMS Microbiol Lett 314:140-146

13. Xiao P, Mori T, Kamei I, Kiyota H, Takagi K, Kondo R (2011) Novel metabolic pathways of organochlorine pesticides dieldrin and aldrin by the white rot fungi of the genus Phlebia. Chemosphere 85:218-224

14. Purnomo AS, Putra SR, Shimizu K, Kondo R (2014) Biodegradation of heptachlor and heptachlor epoxide-contaminated soils by white-rot fungal inocula. Environ Sci Pollut Res 21:11305-11312

15. Xiao P, Kondo R (2019) Biodegradation and bioconversion of endrin by white rot fungi, Phlebia acanthocystis and Phlebia brevispora. Mycoscience 60:255-261

16. Mori T, Kondo R (2002) Degradation of 2,7-dichlorodibenzo-p-dioxin by wood-rotting fungi, screened by dioxin degrading ability. FEMS Microbiol Lett 213(1):127-132

17. Alleman BC, Logan BE, Gilbertson RL (1992) Toxicity of pentachlorophenol to six species of white rot fungi as a function of chemical dose. Appl Environ Microbiol 58(12):4048-4050

18. Kaur H, Kapoor S, Kaur G (2016) Application of ligninolytic potentials of a white-rot fungus Ganoderma lucidum for degradation of lindane. Environ Monit Assess 188:588

19. Mori T, Sudo S, Kawagishi H, Hirai H (2018) Biodegradation of diuron in artificially contaminated water and seawater by wood colonized with the white-rot fungus Trametes versicolor. J Wood Sci 64:690-696

20. Bosso L, Cristinzio G (2014) A comprehensive overview of bacteria and fungi used for pentachlorophenol biodegradation. Rev Environ Sci Biotechnol 13(4):387-427

21. Reddy GVB, Gold MH (2000) Degradation of pentachlorophenol by Phanerochaete chrysosporium: intermediates and reactions involved. Microbiology 146:405-413

22. Cea M, Jorquera M, Rubilar O, Langer H, Tortella G, Diez MC (2010) Bioremediation of soil contaminated with pentachlorophenol by Anthracophyllum discolor and its effect on soil microbial community. J Hazard Mater 181:315-323

23. Zupan N, Babic J, Pavko A (2014) Biodegradation kinetics of phenol, 2,4,6-trichlorophenol and pentachlorophenol with ligninolytic enzymes from Dichomitus squalens. J Adv Chem 8(3):1736-1744 
24. Ning D, Wang H (2012) Involvement of Cytochrome P450 in pentachlorophenol transformation in a white rot fungus Phanerochaete chrysosporium. PLoS ONE 7(9):e45887

25. Tuomela M, Lyytikainen M, Oivanen P, Hatakka A (1999) Mineralization and conversion of pentachlorophenol (PCP) in soil inoculated with the white-rot fungus Trametes versicolor. Soil Biol Biochem 31:65-74

26. Machado KMG, Matheus DR, Monteiro RTR, Bononi VLR (2005) Biodegradation of pentachlorophenol by tropical basidiomycetes in soils contaminated with industrial residues. World J Microbiol Biotechnol 21(3):297-301

\section{Publisher's Note}

Springer Nature remains neutral with regard to jurisdictional claims in published maps and institutional affiliations.

\section{Submit your manuscript to a SpringerOpen ${ }^{\circ}$ journal and benefit from:}

- Convenient online submission

- Rigorous peer review

- Open access: articles freely available online

- High visibility within the field

- Retaining the copyright to your article

Submit your next manuscript at $\boldsymbol{\nabla}$ springeropen.com 